\title{
Direct methods in the calculus of variations for differential forms
}

Tingting Wang*, Gejun Bao and Guanfeng Li

"Correspondence: ttwanghit@gmail.com Department of Mathematics, Harbin Institute of Technology, Harbin, 150001, P.R. China

\begin{abstract}
The purpose of this paper is to establish the general theory of the direct methods to functionals / defined on the Grassmann algebra employing the classical approaches. In this paper, various notions of convexity conditions for weak lower semicontinuity of I are discussed, and existence theorems for minimizers of / are obtained. Lastly, we present some examples to illustrate our main results.
\end{abstract}

MSC: $35 \mathrm{~A} 15 ; 58 \mathrm{~A} 10$

Keywords: weak lower semicontinuity; differential forms; variational problem; direct methods; existence theorem

\section{Introduction}

Differential forms as invaluable tools have been available and applicable to various fields of study, see [1-6], while the systematic investigation of variational theory for differential forms has been less studied. Direct methods is a classical and fundamental method, used in variational problems, which has been widely applied for solving differential equations that possess variational structure, see [7-10]. More precisely, direct methods work for the minimization problem for certain energy functionals $I$, whose critical points are usually related to solutions of certain differential equations. Various conditions for the existence of minimizers of $I$ have been greatly studied, see [9-21].

This paper is intended to generalize and apply the classical direct methods in variational problems for differential forms with an aim to enlarge the range of applications of the variational principle. Our work is motivated by Iwaniec and Lutoborski [1], who first used differential forms to express variational problems.

Let $\Omega$ be a nonempty, bounded, open subset of $\mathbb{R}^{n}$. Suppose that integers $1 \leq l_{1}, \ldots, l_{m} \leq$ $n$ and $W: \wedge^{l_{1}} \times \cdots \times \wedge^{l_{m}} \rightarrow \wedge^{n}$ is a continuous function satisfying the growth condition

$$
\left|W\left(\xi^{1}, \ldots, \xi^{m}\right)\right| \leq C\left(\left|\xi^{1}\right|^{p_{1}}+\cdots+\left|\xi^{m}\right|^{p_{m}}\right)
$$

for all $\xi=\left(\xi^{1}, \ldots, \xi^{m}\right) \in \wedge^{l_{1}} \times \cdots \times \wedge^{l_{m}}$, where $1<p_{1}, \ldots, p_{m}<\infty$. Write $\mathbf{p}=\left(p_{1}, \ldots, p_{m}\right)$. Given $g=\left(g^{1}, \ldots, g^{m}\right) \in W^{1, \mathbf{p}}\left(\Omega, \wedge^{l_{1}} \times \cdots \times \wedge^{l_{m}}\right)$, we consider the integral

$$
I[F]=\int_{\Omega} W(g+d F)=\int_{\Omega} W\left(g^{1}+d f^{1}, \ldots, g^{m}+d f^{m}\right)
$$


defined for vectorial differential forms

$$
F=\left(f^{1}, \ldots, f^{m}\right) \in W^{1, \mathbf{p}}\left(\Omega, \wedge^{l_{1}-1} \times \cdots \times \wedge^{l_{m}-1}\right),
$$

where $\mathbf{p}=\left(p_{1}, \ldots, p_{m}\right)$ is imposed by the growth condition (1.1). Let

$$
\mathcal{M}=\left\{F \mid F \in W_{0}^{1, \mathbf{p}}\left(\Omega, \wedge^{l_{1}-1} \times \cdots \times \wedge^{l_{m}-1}\right)\right\} .
$$

We consider the existence of the minimizer of $I$ in $\mathcal{M}$, that is, we want to prove that

$$
\inf _{\mathcal{M}} I[F]
$$

is achieved.

In particular, associated with the variational kernel $W(\xi)=|\xi|^{p}$, (1.2) is reduced to the $p$-Dirichlet integral

$$
I[u]=\int_{\Omega}|g+d u|^{p},
$$

and the minimizers of the $p$-Dirichlet integral are exactly the weak solutions to the $p$-harmonic equation

$$
d^{*}\left(|g+d u|^{p-2}(g+d u)\right)=0 \text {. }
$$

\section{Notation and preliminary results}

This section is devoted to the notation of the exterior calculus and a few necessary preliminaries. Readers can refer to [1-3] for more details.

We denote by $\wedge^{l}=\wedge^{l}\left(\mathbb{R}^{n}\right)$ the space of $l$-covectors in $\mathbb{R}^{n}$, and the direct sum

$$
\wedge\left(\mathbb{R}^{n}\right)=\bigoplus_{l=0}^{n} \wedge^{l}\left(\mathbb{R}^{n}\right)
$$

is a graded algebra with respect to the wedge product $\wedge$. We shall make use of the exterior derivative

$$
d: C^{\infty}\left(\Omega, \wedge^{l}\right) \rightarrow C^{\infty}\left(\Omega, \wedge^{l+1}\right)
$$

and its formal adjoint operator

$$
d^{*}=(-1)^{n l+1} * d *: C^{\infty}\left(\Omega, \wedge^{l+1}\right) \rightarrow C^{\infty}\left(\Omega, \wedge^{l}\right),
$$

known as the Hodge codifferential, where the symbol $*$ denotes the Hodge star duality operator. Note that each of the operators $d$ and $d^{*}$ applied twice gives a zero.

Let $C^{\infty}\left(\bar{\Omega}, \wedge^{l}\right)$ be the class of infinitely differentiable $l$-forms on $\bar{\Omega} \subset \mathbb{R}^{n}$. Since $\Omega$ is a smooth domain, near each boundary point one can introduce a local coordinate system $\left(x_{1}, x_{2}, \ldots, x_{n}\right)$ such that $x_{n}=0$ on $\partial \Omega$, and such that the $x_{n}$-curve is orthogonal to $\partial \Omega$. 
Near this boundary point, every differential form $\omega \in C^{\infty}\left(\bar{\Omega}, \wedge^{l}\right)$ can be decomposed as $\omega(x)=\omega_{T}(x)+\omega_{N}(x)$, where

$$
\omega_{T}(x)=\sum_{1 \leq i_{1}<\cdots<i_{l}<n} \omega_{i_{1}, \ldots, i_{l}}(x) d x_{i_{1}} \wedge \cdots \wedge d x_{i_{l}}
$$

and

$$
\omega_{N}(x)=\sum_{1 \leq i_{1}<\cdots<i_{l}=n} \omega_{i_{1}, \ldots, i_{l}}(x) d x_{i_{1}} \wedge \cdots \wedge d x_{i_{l}}
$$

are called the tangential and the normal part of $\omega$, respectively. Now, the duality between $d$ and $d^{*}$ is expressed by the integration by parts formula

$$
\int_{\Omega}\langle d u, v\rangle=\int_{\Omega}\left\langle u, d^{*} v\right\rangle
$$

for all $u \in C^{\infty}\left(\bar{\Omega}, \wedge^{l}\right)$ and $v \in C^{\infty}\left(\bar{\Omega}, \wedge^{l+1}\right)$, provided $u_{T}=0$ or $v_{N}=0$. The symbol $\langle\cdot, \cdot\rangle$ denotes the inner product, i.e., let $\alpha=\sum_{I} \alpha_{I}(x) d x_{I}$ and $\beta=\sum_{I} \beta_{I}(x) d x_{I}$, then $\langle\alpha, \beta\rangle=$ $\sum_{I} \alpha_{I}(x) \beta_{I}(x)$.

Due to (2.1), extended definitions for $d$ and $d^{*}$ can be introduced as the introduction of weak derivatives.

Definition 2.1 [2] Suppose that $\omega \in L_{\mathrm{loc}}^{1}\left(\Omega, \wedge^{l}\right)$ and $v \in L_{\mathrm{loc}}^{1}\left(\Omega, \wedge^{l+1}\right)$. If

$$
\int_{\Omega}\left\langle\omega, d^{*} \eta\right\rangle=\int_{\Omega}\langle v, \eta\rangle
$$

for every test form $\eta \in C_{0}^{\infty}\left(\Omega, \wedge^{l+1}\right)$, we say that $\omega$ has generalized exterior derivative $v$ and write $v=\tilde{d} \omega$.

The notion of the generalized exterior coderivative $\tilde{d}^{*}$ can be defined analogously.

Definition 2.2 [2] Suppose that $\omega \in L_{\mathrm{loc}}^{1}\left(\Omega, \wedge^{l}\right)$ and $v \in L_{\mathrm{loc}}^{1}\left(\Omega, \wedge^{l-1}\right)$. If

$$
\int_{\Omega}\langle\omega, d \eta\rangle=\int_{\Omega}\langle v, \eta\rangle
$$

for every test form $\eta \in C_{0}^{\infty}\left(\Omega, \wedge^{l-1}\right)$, we say that $\omega$ has generalized exterior coderivative $v$ and write $v=\tilde{d}^{*} \omega$.

Remark [6] (i) The generalized exterior derivative has many properties similar to those of the weak derivative. For example, $\left(i_{1}\right)$ if the generalized exterior derivative exists, it is unique; $\left(i_{2}\right)$ if $\omega$ is differentiable in the conventional sense, then its generalized exterior derivative $\tilde{d} \omega$ is identical to its classical exterior differential $d \omega$. Analogous results hold for generalized exterior coderivative.

(ii) If the generalized exterior derivative of $\omega, \tilde{d} \omega$, exists, then $\tilde{d} \omega$ also has its generalized exterior derivative $\tilde{d}(\tilde{d} \omega)$. Moreover, $\tilde{d}(\tilde{d} \omega)=0$. 
(iii) $d$ and $\tilde{d}$ have analogous expressions, i.e., for $\omega(x)=\sum_{I} \omega_{I}(x) d x_{I}$, we have

$$
d \omega(x)=\sum_{I} \sum_{k=1}^{n} \frac{\partial \omega_{I}}{\partial x_{k}} d x_{k} \wedge d x_{I}
$$

and

$$
\tilde{d} \omega(x)=\sum_{I} \sum_{k=1}^{n} \frac{\tilde{\partial} \omega_{I}}{\partial x_{k}} d x_{k} \wedge d x_{I}
$$

where $\partial$ denotes the ordinary derivative and $\tilde{\partial}$ the weak derivative. So next, we use $d$ to represent the action instead of $\tilde{d}$, similar for $d^{*}$ and $\tilde{d}^{*}$.

Next, we present briefly some spaces of differential forms

$$
\begin{aligned}
& L^{q}\left(\Omega, \wedge^{l}\right) \text {-the space of } l \text { - forms } \omega \text { with coefficients in } L^{q}(\Omega) ; \\
& L_{1}^{q}\left(\Omega, \wedge^{l}\right) \text { - the space of } l \text {-forms } \omega \text { such that } \nabla \omega \text { is a regular } \\
& \text { distribution of } L^{q}\left(\Omega, \wedge^{l}\right) \text {; } \\
& W^{1, q}\left(\Omega, \wedge^{l}\right) \text { - the Sobolev space of } l \text {-forms } \omega \text { defined by } L^{q}\left(\Omega, \wedge^{l}\right) \cap L_{1}^{q}\left(\Omega, \wedge^{l}\right),
\end{aligned}
$$

which are used throughout this paper.

Finally, we introduce the definition of weak convergence for sequences in spaces of differential forms as needed. Throughout the paper, let $1<q, q^{\prime}<\infty$ be the Hölder conjugate pair, $q+q^{\prime}=q q^{\prime}$.

Definition 2.3 [6] We say that $\varphi_{j}$ weakly converges to $\varphi$ in $L^{q}\left(\Omega, \wedge^{l}\right)$ if

$$
\int_{\Omega} \varphi_{j} \wedge h \rightarrow \int_{\Omega} \varphi \wedge h
$$

whenever $h \in L^{q^{\prime}}\left(\Omega, \wedge^{n-l}\right)$ and write $\varphi_{j} \rightarrow \varphi$ in $L^{q}\left(\Omega, \wedge^{l}\right)$.

We also need some properties of spaces of differential forms.

Proposition 2.4 [6] For $1<q<\infty, L^{q}\left(\Omega, \wedge^{l}\right)$ is reflexive.

Proposition $2.5[6] \varphi_{j} \rightarrow \varphi$ in $W^{1, q}\left(\Omega, \wedge^{l}\right)$ if and only if $\varphi_{j} \rightarrow \varphi$ in $L^{q}\left(\Omega, \wedge^{l}\right)$ and $\nabla \varphi_{j} \rightarrow$ $\nabla \varphi$ in $L^{q}\left(\Omega, \wedge^{l}\right)$, where $\nabla \varphi=\left(\frac{\partial \varphi}{\partial x_{1}}, \ldots, \frac{\partial \varphi}{\partial x_{n}}\right)$, and the partial differentiation is applied to the coefficients of $\varphi$.

\section{Weak lower semicontinuity}

In many variational problems, weak lower semicontinuity is an essential condition for the existence of minimizers, using the minimization method. This will motivate the study of various notions of convexity conditions. In this section, we study the conditions for weak lower semicontinuity of the integral functional $I[F]$ in the Sobolev space $W^{1, \mathbf{p}}\left(\Omega, \wedge^{l_{1}-1} \times\right.$ $\cdots \times \wedge^{l_{m-1}}$ ). 
Definition 3.1 $I$ is called weak lower semicontinuous on $W^{1, \mathbf{p}}\left(\Omega, \wedge^{l_{1}-1} \times \cdots \times \wedge^{l_{m}-1}\right)$ if

$$
I[F] \leq \liminf _{v \rightarrow \infty} I\left[F_{v}\right]
$$

whenever $F_{v}=\left(f_{v}^{1}, \ldots, f_{v}^{m}\right) \rightarrow F=\left(f^{1}, \ldots, f^{m}\right)$ in $W^{1, \mathbf{p}}\left(\Omega, \wedge^{l_{1}-1} \times \cdots \times \wedge^{l_{m}-1}\right)$.

\subsection{The convex case}

Theorem 3.2 For a given $g=\left(g^{1}, \ldots, g^{m}\right) \in W^{1, \mathbf{p}}\left(\Omega, \wedge^{l_{1}} \times \cdots \times \wedge^{l_{m}}\right)$, let $W \geq 0$ be continuous and convex. Then $I$ is weakly lower semicontinuous on $W^{1, \mathbf{p}}\left(\Omega, \wedge^{l_{1}-1} \times \cdots \times \wedge^{l_{m}-1}\right)$.

Proof Suppose that $F_{v}=\left(f_{v}^{1}, \ldots, f_{v}^{m}\right) \rightarrow F=\left(f^{1}, \ldots, f^{m}\right)$ in $W^{1, \mathbf{p}}\left(\Omega, \wedge^{l_{1}-1} \times \cdots \times \wedge^{l_{m}-1}\right)$, we then need to show

$$
I[F] \leq \liminf _{\nu \rightarrow \infty} I\left[F_{v}\right]
$$

We may assume that $\left\{I\left[F_{v}\right]\right\}$ is finite and convergent.

Since $d F_{v} \rightarrow d F$ in $L^{\mathbf{P}}\left(\Omega, \wedge^{l_{1}} \times \cdots \times \wedge^{l_{m}}\right)$, then, according to Mazur lemma [22], for any $v_{0} \in \mathbb{N}$, there exists a sequence $\left\{\mathcal{F}^{l}\right\}_{l \geq v_{0}}$ of convex linear combinations

$$
\mathcal{F}^{l}=\sum_{\nu=\nu_{0}}^{l} \lambda_{\nu}^{l} d F_{\nu}, \quad 0 \leq \lambda_{\nu}^{l} \leq 1, \quad \sum_{\nu=\nu_{0}}^{l} \lambda_{v}^{l}=1, \quad l \geq v_{0}
$$

such that $\mathcal{F}^{l} \rightarrow d F(l \rightarrow \infty)$ in $L^{\mathbf{P}}\left(\Omega, \wedge^{l_{1}} \times \cdots \times \wedge^{l_{m}}\right)$ and pointwise almost everywhere.

Since $W$ is continuous, we have

$$
W\left(g+\mathcal{F}_{l}\right) \rightarrow W(g+d F)
$$

as $l \rightarrow \infty$. On the other hand, we have by the convexity of $W$ that

$$
\begin{aligned}
W\left(g+\mathcal{F}_{l}\right) & =W\left(\sum_{\nu=\nu_{0}}^{l} \lambda_{v}^{l}\left(g+d F_{v}\right)\right) \\
& \leq \sum_{\nu=\nu_{0}}^{l} \lambda_{\nu}^{l} W\left(g+d F_{v}\right) .
\end{aligned}
$$

Therefore, we obtain from Fatou lemma that

$$
\begin{aligned}
\int_{\Omega} W(g+d F) & =\int_{\Omega} \lim _{l \rightarrow \infty} W\left(g+\mathcal{F}^{l}\right) \\
& =\int_{\Omega} \liminf _{l \rightarrow \infty} W\left(g+\mathcal{F}^{l}\right) \\
& \leq \liminf _{l \rightarrow \infty} \int_{\Omega} W\left(g+\mathcal{F}^{l}\right) \\
& \leq \liminf _{l \rightarrow \infty} \sum_{\nu=\nu_{0}}^{l} \lambda_{\nu}^{l} \int_{\Omega} W\left(g+d F_{v}\right)
\end{aligned}
$$




$$
\begin{aligned}
& \leq \liminf _{l \rightarrow \infty} \sup _{v_{0} \leq v \leq l} \int_{\Omega} W\left(g+d F_{v}\right) \\
& =\liminf _{l \rightarrow \infty} \sup _{k \geq l_{\nu_{0} \leq \nu \leq k}} \int_{\Omega} W\left(g+d F_{v}\right) \\
& =\lim _{l \rightarrow \infty} \sup _{\nu_{0} \leq \nu \leq l} \int_{\Omega} W\left(g+d F_{\nu}\right) \\
& =\sup _{\nu \geq \nu_{0}} \int_{\Omega} W\left(g+d F_{v}\right) .
\end{aligned}
$$

Let $v_{0} \rightarrow \infty$ in the inequality above, we obtain

$$
\begin{aligned}
\int_{\Omega} W(g+d F) & \leq \lim _{\nu_{0} \rightarrow \infty} \sup _{\nu \geq v_{0}} \int_{\Omega} W\left(g+d F_{\nu}\right) \\
& =\limsup _{\nu \rightarrow \infty} \int_{\Omega} W\left(g+d F_{v}\right) \\
& =\liminf _{\nu \rightarrow \infty} \int_{\Omega} W\left(g+d F_{\nu}\right),
\end{aligned}
$$

that is, $I[F] \leq \liminf _{v \rightarrow \infty} I\left[F_{v}\right]$. The theorem follows.

\subsection{The non-convex case}

This section considers the case when $W$ is not convex. Fruitful results have been obtained for the weak lower semicontinuity of integral functionals $I$ when $W$ fails to be convex, see [9-21]. We consider the counterpart in the case of differential forms.

\subsubsection{Quasiconvex}

Definition 3.3 Let $W: \wedge^{l_{1}} \times \cdots \times \wedge^{l_{m}} \rightarrow \wedge^{n}$ be continuous. Then $W$ is said to be quasiconvex if

$$
\int_{D} W\left(\xi_{0}+d \phi(x)\right) \geq \int_{D} W\left(\xi_{0}\right)
$$

holds for every bounded open set $D \subset \mathbb{R}^{n}$ with mea $\partial D=0$ for every fixed $\xi_{0} \in \wedge^{l_{1}} \times \cdots \times$ $\wedge^{l_{m}}$ and for all $\phi \in W_{0}^{1, \mathbf{p}}\left(\Omega, \wedge^{l_{1}-1} \times \cdots \times \wedge^{l_{m}-1}\right)$.

Remark (i) This definition is actually the so-called $W^{1, p}$-quasiconvex, see [14]. For the general notion of quasiconvex (relatively is $W^{1, \infty}$-quasiconvex) and other results, readers can refer to $[9,10,13,16]$ and references therein.

(ii) From Jensen's inequality, we see that every convex function is quasiconvex. In fact, we have by Jensen's inequality that

$$
\begin{aligned}
\frac{1}{|\Omega|} \int_{\Omega} W\left(\xi_{0}+d \phi(x)\right) & \geq W\left(\frac{1}{|\Omega|} \int_{\Omega} \xi_{0}+d \phi(x)\right) \\
& =W\left(\xi_{0}+\frac{1}{|\Omega|} \int_{\Omega} d \phi(x)\right) .
\end{aligned}
$$

Since $\phi \in W_{0}^{1, \mathbf{p}}\left(\Omega, \wedge^{l_{1}-1} \times \cdots \times \wedge^{l_{m}-1}\right)$, the Stokes theorem [4] yields

$$
\int_{\Omega} d \phi(x)=\int_{\partial \Omega} \phi(x)=0 .
$$


Therefore, we have

$$
\frac{1}{|\Omega|} \int_{\Omega} W\left(\xi_{0}+d \phi(x)\right) \geq W\left(\xi_{0}\right)
$$

(iii) Quasiconvexity implies that the function $W$ is convex with respect to each $\xi^{i} \in \wedge^{l_{i}}$, $i=1, \ldots, m$, which together with (1.1) yields a Lipschitz-type condition

$$
|W(\xi+\zeta)-W(\xi)| \leq C(\mathbf{p}) \sum_{i=1}^{m}\left|\zeta^{i}\right|\left(\left|\xi^{i}\right|+\left|\zeta^{i}\right|\right)^{p_{i}-1}
$$

Lemma 3.4 Let $\xi_{0} \in \wedge^{l_{1}} \times \cdots \times \wedge^{l_{m}}$ and $W$ be quasiconvex satisfying the growth condition (1.1). Suppose that

$$
F_{v}=\left(f_{v}^{1}, \ldots, f_{v}^{m}\right) \rightarrow 0
$$

in $W^{1, \mathbf{p}}\left(\Omega, \wedge^{l_{1}-1} \times \cdots \times \wedge^{l_{m}-1}\right)$. Then

$$
\liminf _{\nu \rightarrow \infty} \int_{\Omega} W\left(\xi_{0}+d F_{\nu}\right) \geq \int_{\Omega} W\left(\xi_{0}\right)
$$

Proof Let $\Omega_{v}=\{x \in \Omega: \operatorname{dist}(x, \partial \Omega)>1 / v\}$, then $\left|\Omega-\Omega_{v}\right| \rightarrow 0$ as $v \rightarrow \infty$. We choose cut-off functions $\eta_{v} \in C_{0}^{\infty}(\Omega)$ such that $0 \leq \eta_{v} \leq 1,\left.\eta_{v}\right|_{\Omega_{v}}=1$ and $\left|\nabla \eta_{v}\right| \leq C_{v}<\infty$. Let

$$
\phi_{v}(x)=\eta_{v}(x) F_{v}(x)
$$

then $\phi_{v} \in W_{0}^{1, \mathbf{p}}\left(\Omega, \wedge^{l_{1}-1} \times \cdots \times \wedge^{l_{m}-1}\right)$ and

$$
d \phi_{v}(x)=d \eta_{v}(x) \wedge F_{v}(x)+\eta_{v}(x) d F_{v}(x)
$$

We take $\phi_{v}$ as test functions in the definition of quasiconvexity to obtain

$$
\begin{aligned}
\int_{\Omega} W\left(\xi_{0}\right) & \leq \int_{\Omega} W\left(\xi_{0}+d \phi_{\nu}(x)\right) \\
& =\int_{\Omega_{v}} W\left(\xi_{0}+d F_{v}(x)\right)+\int_{\Omega_{\backslash \Omega_{v}}} W\left(\xi_{0}+d \phi_{\nu}(x)\right) \\
& =\int_{\Omega} W\left(\xi_{0}+d F_{v}(x)\right)+\int_{\Omega \backslash \Omega_{v}}\left(W\left(\xi_{0}+d \phi_{\nu}(x)\right)-W\left(\xi_{0}+d F_{v}(x)\right)\right) .
\end{aligned}
$$

Since $W$ is continuous, thus, $W$ is bounded on bounded sets, together with that $\mid \Omega-$ $\Omega_{v} \mid \rightarrow 0$ as $v \rightarrow \infty$, we then have the last integral in the right hand side of (3.4) converges to zero as $v \rightarrow \infty$. Therefore, we have

$$
\int_{\Omega} W\left(\xi_{0}\right) \leq \liminf _{\nu \rightarrow \infty} \int_{\Omega} W\left(\xi_{0}+d F_{v}\right)
$$

which is the desired result.

Theorem 3.5 Let $W \geq 0$ be quasiconvex and satisfy (1.1). Then I is weakly lower semicontinuous in $W^{1, \mathbf{p}}\left(\Omega, \wedge^{l_{1}-1} \times \cdots \times \wedge^{l_{m}-1}\right)$. 
Proof Suppose that $F_{v}=\left(f_{v}^{1}, \ldots, f_{v}^{m}\right) \rightarrow F=\left(f^{1}, \ldots, f^{m}\right)$ in $W^{1, \mathbf{p}}\left(\Omega, \wedge^{l_{1}-1} \times \cdots \times \wedge^{l_{m}-1}\right)$, we then need to show that

$$
I[F] \leq \liminf _{\nu \rightarrow \infty} I\left[F_{v}\right]
$$

We may assume that $\left\{I\left[F_{v}\right]\right\}$ is finite and convergent, i.e.,

$$
\liminf _{\nu \rightarrow \infty} I\left[F_{\nu}\right]=\lim _{\nu \rightarrow \infty} I\left[F_{\nu}\right]
$$

Let $Q_{k} \subset \Omega$ be disjoint cubes parallel to the axes, and edge-length is $1 / k$. Denote $\Omega_{k}=$ $\bigcup Q_{k}$, and we then have that

$$
\left|\Omega-\Omega_{k}\right| \rightarrow 0
$$

as $k \rightarrow \infty$. Let

$$
d F(x)=\sum_{I}(d F)_{I}(x) d x_{I}
$$

with $(d F)_{I}(x) \in L^{\mathbf{P}}\left(\Omega, \wedge^{l_{1}} \times \cdots \times \wedge^{l_{m}}\right)$. For $x \in Q_{k}$, set

$$
\left(\xi_{k}\right)_{I}=\frac{1}{\left|Q_{k}\right|} \int_{Q_{k}}(d F)_{I}(y) d y
$$

Then $\left(\xi_{k}\right)_{I}$ is constant on $Q_{k}$ and write $\xi_{k}=\sum_{I}\left(\xi_{k}\right)_{I} d x_{I} \in \wedge^{l_{1}} \times \cdots \times \wedge^{l_{m}}$. Then we have

$$
\left\|d F(x)-\xi_{k}\right\|_{p, Q_{k}}=\left(\sum_{I} \int_{Q_{k}}\left|(d F)_{I}(x)-\left(\xi_{k}\right)_{I}\right|^{p} d x\right)^{\frac{1}{p}} \longrightarrow 0
$$

as $k \rightarrow \infty$. Therefore, for arbitrary $\varepsilon>0$, we can choose $k$ large enough such that

$$
\left|\Omega-\Omega_{k}\right|<\varepsilon \quad \text { and } \quad\left\|d F(x)-\xi_{k}\right\|_{p, Q_{k}}<\varepsilon
$$

Note that $F_{v}=\left(f_{v}^{1}, \ldots, f_{v}^{m}\right) \rightarrow F=\left(f^{1}, \ldots, f^{m}\right)$ in $W^{1, \mathbf{p}}\left(\Omega, \wedge^{l_{1}-1} \times \cdots \times \wedge^{l_{m}-1}\right)$, we have $\left\|F_{v}\right\|_{1, \mathbf{p}, \Omega}$ is uniformly bounded. We then consider

$$
\begin{aligned}
I\left[F_{\nu}\right]-I[F] & =\int_{\Omega}\left(W\left(g+d F_{v}\right)-W(g+d F)\right) \\
& \triangleq J_{1}+J_{2}+J_{3}+J_{4},
\end{aligned}
$$

where

$$
\begin{aligned}
& J_{1}=\int_{\Omega \backslash \Omega_{k}}\left(W\left(g+d F_{v}\right)-W(g+d F)\right), \\
& J_{2}=\sum_{k} \int_{Q_{k}}\left(W\left(g+d F_{v}\right)-W\left(g+\xi_{k}+d F_{v}-d F\right)\right),
\end{aligned}
$$




$$
\begin{aligned}
& J_{3}=\sum_{k} \int_{Q_{k}}\left(W\left(g+\xi_{k}+d F_{v}-d F\right)-W\left(g+\xi_{k}\right)\right), \\
& J_{4}=\sum_{k} \int_{Q_{k}}\left(W\left(g+\xi_{k}\right)-W(g+d F)\right) .
\end{aligned}
$$

For $J_{1}$ : Since $W \geq 0, W$ is continuous and $\Omega$ is bounded, we have

$$
\begin{aligned}
J_{1} & =\int_{\Omega \backslash \Omega_{k}}\left(W\left(g+d F_{v}\right)-W(g+d F)\right) \geq-\int_{\Omega \backslash \Omega_{k}} W(g+d F) \\
& \geq-\sup _{\Omega}|W| \cdot\left|\Omega-\Omega_{k}\right| \\
& \geq-\varepsilon \sup _{\Omega}|W|=-\varepsilon C_{1} ;
\end{aligned}
$$

For $J_{2}$ : From (3.2) and the Hölder inequality, we see that

$$
\begin{aligned}
\left|J_{2}\right| & \leq \sum_{k} \int_{Q_{k}}\left|W\left(g+d F_{v}\right)-W\left(g+\xi_{k}+d F_{v}-d F\right)\right| \\
& \leq C(\mathbf{p}) \sum_{k} \sum_{i=1}^{m} \int_{Q_{k}}\left|d f^{i}-\xi_{k}^{i}\right|\left(\left|g^{i}+d f_{v}^{i}\right|+\left|d f^{i}-\xi_{k}^{i}\right|\right)^{p_{i}-1} \\
& \leq C(\mathbf{p}) \sum_{k} \sum_{i=1}^{m}\left(\int_{Q_{k}}\left|d f^{i}-\xi_{k}^{i}\right|\left|g^{i}\right|^{p_{i}-1}+\left|d f^{i}-\xi_{k}^{i}\right|\left|d f_{v}^{i}\right|^{p_{i}-1}+\int_{Q_{k}}\left|d f^{i}-\xi_{k}^{i}\right|^{p_{i}}\right) \\
& \leq C(\mathbf{p}, m)\left(\|g\|_{\mathbf{p}, Q_{k}}^{\mathbf{p}-1}+\left\|d F_{v}\right\|_{\mathbf{p}, Q_{k}}^{\mathbf{p}-1}\right)\left\|d F-\xi_{k}\right\|_{\mathbf{p}, Q_{k}}+\left\|d F-\xi_{k}\right\|_{\mathbf{p}, Q_{k}}^{\mathbf{p}} \\
& \leq \varepsilon C_{2} ;
\end{aligned}
$$

For $J_{3}$ : Applying Lemma 3.4 for $\xi_{0}=g+\xi_{k}$, yields

$$
\liminf _{\nu \rightarrow \infty} \int_{Q_{k}} W\left(g+\xi_{k}+d F_{\nu}-d F\right) \geq \int_{Q_{k}} W\left(g+\xi_{k}\right),
$$

which implies that

$$
\liminf _{\nu \rightarrow \infty} J_{3} \geq 0
$$

For $J_{4}$ : It follows from (3.2) and the Hölder inequality that

$$
\begin{aligned}
\left|J_{4}\right| & =\sum_{k} \int_{Q_{k}}\left|W\left(g+\xi_{k}\right)-W(g+d F)\right| \\
& \leq C(\mathbf{p}) \sum_{k} \sum_{i=1}^{m} \int_{Q_{k}}\left|d f^{i}-\xi_{k}^{i}\right|\left(\left|g^{i}\right|+\left|d f^{i}\right|+\left|\xi_{k}^{i}\right|\right)^{p_{i}-1} \\
& \leq C(\mathbf{p}, m)\left(\|g\|_{\mathbf{p}, Q_{k}}^{\mathbf{p}-1}+\|d F\|_{\mathbf{p}, Q_{k}}^{\mathbf{p}-1}+\left\|\xi_{k}\right\|_{\mathbf{p}, Q_{k}}^{\mathbf{p}-1}\right)\left\|d F-\xi_{k}\right\|_{\mathbf{p}, Q_{k}} \\
& \leq \varepsilon C_{3} .
\end{aligned}
$$

Substituting (3.6)-(3.9) into (3.5), we have by letting $v \rightarrow \infty$ that

$$
\liminf _{\nu \rightarrow \infty} I\left[F_{\nu}\right]-I[F] \geq-\varepsilon\left(C_{1}+C_{2}+C_{3}\right) .
$$


Let $\varepsilon \rightarrow 0$ in the above inequality, we obtain

$$
\liminf _{\nu \rightarrow \infty} I\left[F_{v}\right] \geq I[F]
$$

The theorem follows.

\subsubsection{Polyconvex}

Polyconvexity is also an important condition for the weak lower semicontinuity of $I$, which is quasiconvex, but not necessarily convex. We give below the definition of polyconvex, and we want to establish the weakly lower semicontinuity of $I$ on $W^{1, \mathbf{p}}\left(\Omega, \wedge^{l_{1}-1} \times \cdots \times\right.$ $\left.\wedge^{l_{m}-1}\right)$, provided $W$ is polyconvex.

Definition 3.6 Let $N=N(m)$ be the number of elements of the set

$$
T(\xi)=\left\{\xi^{i_{1}} \wedge \cdots \wedge \xi^{i_{k}} \mid 1 \leq i_{1}<\cdots<i_{k} \leq m, k=1, \ldots, m\right\}
$$

for $\xi=\left(\xi^{1}, \ldots, \xi^{m}\right) \in \wedge^{l_{1}} \times \cdots \times \wedge^{l_{m}}$. A function $W: \wedge^{l_{1}} \times \cdots \times \wedge^{l_{m}} \rightarrow \wedge^{n}$ is called polyconvex if there exists $g: \mathbb{R}^{N} \rightarrow \wedge^{n}$ be convex in each of its variables, such that

$$
W(\xi)=g(T(\xi))
$$

Proceeding with the proof in much the same way as [9], the equivalent definition for polyconvexity is given by Iwaniec and Lutoborski.

Definition 3.7 [1] A continuous function $W: \wedge^{l_{1}} \times \cdots \times \wedge^{l_{m}} \rightarrow \wedge^{n}$ is called polyconvex if

$$
W(\xi)-W(\zeta) \geq \sum_{k=1}^{m} \sum_{1 \leq i_{1}<\cdots<i_{k} \leq m} A_{i_{1} \cdots i_{k}}(\zeta) \wedge\left(\xi^{i_{1}} \wedge \cdots \wedge \xi^{i_{k}}-\zeta^{i_{1}} \wedge \cdots \wedge \zeta^{i_{k}}\right)
$$

where $\xi=\left(\xi^{1}, \ldots, \xi^{m}\right)$ and $\zeta=\left(\zeta^{1}, \ldots, \zeta^{m}\right)$ are variable points from $\wedge^{l_{1}} \times \cdots \times \wedge^{l_{m}}$ and $A_{i_{1} \cdots i_{k}}: \wedge^{l_{1}} \times \cdots \times \wedge^{l_{m}} \rightarrow \wedge^{n-l}, l=l_{i_{1}}+\cdots+l_{i_{k}}$, are functions of $\zeta$ only.

Remark (i) Note that (3.10) implies convexity of $W$ in each variable, and we have from the definitions that

$$
\text { Convex } \Rightarrow \text { Polyconvex } \Rightarrow \text { Quasiconvex. }
$$

(ii) The non-zero terms in the right side of (3.10) imply that

$$
k \leq m \leq l_{1}+\cdots+l_{m} \leq n .
$$

(iii) For $F=\left(f^{1}, \ldots, f^{m}\right) \in W^{1, \mathbf{p}}\left(\Omega, \wedge^{l_{1}-1} \times \cdots \times \wedge^{l_{m}-1}\right)$, and assume that

$$
f^{i_{j}}=\sum_{I}\left(f^{i_{j}}\right)_{I} d x_{I}, \quad j=1, \ldots, k
$$


then

$$
\begin{aligned}
d f^{i_{1}} & \wedge \cdots \wedge d f^{i_{k}} \\
& =\left(\sum_{I} \sum_{j=1}^{n} \frac{\partial\left(f^{i_{1}}\right)_{I}}{\partial x_{j}} d x_{j} \wedge d x_{I}\right) \wedge \cdots \wedge\left(\sum_{I} \sum_{j=1}^{n} \frac{\partial\left(f^{i_{k}}\right)_{I}}{\partial x_{j}} d x_{j} \wedge d x_{I}\right) \\
& =\sum_{\substack{I_{\nu_{1}}, \ldots, I_{v_{k}} \\
j_{1}, \ldots, j_{k}}}(-1)^{\Delta} \frac{\partial\left(\left(f^{i_{1}}\right)_{I_{\nu_{1}}}, \ldots,\left(f^{i_{k}}\right)_{I_{v_{k}}}\right)}{\partial\left(x_{j_{1}}, \ldots, x_{j_{k}}\right)} d x_{j_{1}} \wedge d x_{I_{\nu_{1}}} \wedge \cdots \wedge d x_{j_{k}} \wedge d x_{I_{v_{k}}},
\end{aligned}
$$

where $(-1)^{\triangle}=1$ or $(-1)$. Observe that the coefficients of (3.11) are $k \times k$ subdeterminant of the Jacobian of $f: \Omega \rightarrow \mathbb{R}^{N}, N=C_{n}^{l_{1}-1}+\cdots+C_{n}^{l_{m}-1}$, where

$$
f=\left(\left(f^{1}\right)_{I_{1}}, \ldots,\left(f^{1}\right)_{I_{C_{n}^{l_{1}-1}}}, \ldots,\left(f^{m}\right)_{I_{1}}, \ldots,\left(f^{m}\right)_{I_{C_{n} l_{m-1}}}\right) .
$$

We apply the following lemma.

Lemma 3.8 [16, Corollary 5.32] Let $\mathcal{J}_{k}(D u)$ be any $k \times k$ subdeterminant, and let $q>k$ be a number. Let $\left\{u_{j}\right\}$ be any sequence weakly convergent to $\bar{u}$ in $W^{1, q}\left(\Omega, \mathbb{R}^{N}\right)$ as $j \rightarrow \infty$. Then $\mathcal{J}_{k}\left(D u_{j}\right) \rightarrow \mathcal{J}_{k}(D \bar{u})$ weakly in $L^{\frac{q}{k}}(\Omega)$.

By adding the condition

$$
\tilde{p} \triangleq \min \left\{p_{1}, \ldots, p_{m}\right\}>m
$$

we have that for $s=1, \ldots, m$,

$$
d f_{v}^{i_{1}} \wedge \cdots \wedge d f_{v}^{i_{s}} \rightarrow d f^{i_{1}} \wedge \cdots \wedge d f^{i_{s}}
$$

in $L^{\frac{\tilde{p}}{s}}\left(\Omega, \wedge^{l_{i_{1}}} \times \cdots \times \wedge^{l_{i_{s}}}\right)$ as $v \rightarrow \infty$ when $F_{v} \rightarrow F$ in $W^{1, \mathbf{p}}\left(\Omega, \wedge^{l_{1}-1} \times \cdots \times \wedge^{l_{m}-1}\right)$. Therefore, we obtain the following theorem for the weakly lower semicontinuity of $I$.

Theorem 3.9 Let $W \geq 0$ be polyconvex, and let $\tilde{p} \triangleq \min \left\{p_{1}, \ldots, p_{m}\right\}>m$. Then I is weakly lower semicontinuous, provided functions $A_{i_{1} \cdots i_{k}}$ in (3.10) belong to $L^{\frac{\tilde{p}}{\tilde{p}-m}}\left(\Omega, \wedge^{n-l}\right)$.

Proof Suppose that $F_{v}=\left(f_{v}^{1}, \ldots, f_{v}^{m}\right) \rightarrow F=\left(f^{1}, \ldots, f^{m}\right)$ in $W^{1, \mathbf{p}}\left(\Omega, \wedge^{l_{1}-1} \times \cdots \times \wedge^{l_{m}-1}\right)$. We may assume that $\left\{I\left[F_{v}\right]\right\}$ is finite and convergent as before. We first obtain from (3.10) that

$$
\begin{aligned}
W\left(g+d F_{v}\right)-W(g+d F) & \\
\geq & \sum_{\substack{1 \leq k \leq m \\
1 \leq i_{1}<\cdots<i_{k} \leq m}} A_{i_{1} \cdots i_{k}}(g+d F) \\
& \wedge\left(\left(g^{i_{1}}+d f_{\nu}^{i_{1}}\right) \wedge \cdots \wedge\left(g^{i_{k}}+d f_{v}^{i_{k}}\right)-\left(g^{i_{1}}+d f^{i_{1}}\right) \wedge \cdots \wedge\left(g^{i_{k}}+d f^{i_{k}}\right)\right) \\
= & \sum_{\substack{1 \leq k \leq m \\
1 \leq i_{1}<\cdots<i_{k} \leq m}} A_{i_{1} \cdots i_{k}} \wedge\left(d f_{v}^{i_{1}} \wedge \cdots \wedge d f_{v}^{i_{k}}-d f^{i_{1}} \wedge \cdots \wedge d f^{i_{k}}\right)
\end{aligned}
$$




$$
\begin{aligned}
& +\sum_{\substack{1 \leq k \leq m \\
1 \leq i_{1}<\cdots<i_{k} \leq m}} A_{i_{1} \cdots i_{k}} \wedge g^{i_{1}} \wedge\left(d f_{v}^{i_{2}} \wedge \cdots \wedge d f_{v}^{i_{k}}-d f^{i_{2}} \wedge \cdots \wedge d f^{i_{k}}\right) \\
& +\sum_{\substack{1 \leq k \leq m \\
1 \leq i_{1}<\cdots<i_{k} \leq m}} A_{i_{1} \cdots i_{k}} \wedge g^{i_{1}} \wedge g^{i_{2}} \wedge\left(d f_{v}^{i_{3}} \wedge \cdots \wedge d f_{v}^{i_{k}}-d f^{i_{3}} \wedge \cdots \wedge d f^{i_{k}}\right)+\cdots \\
& +\sum_{\substack{1 \leq k \leq m \\
1 \leq i_{1}<\cdots<i_{k} \leq m}} A_{i_{1} \cdots i_{k}} \wedge g^{i_{1}} \wedge \cdots \wedge g^{i_{k-1}} \wedge\left(d f_{v}^{i_{k}}-d f^{i_{k}}\right) .
\end{aligned}
$$

Next, observe that for $k=1, \ldots, m$,

$$
d f_{v}^{i_{1}} \wedge \cdots \wedge d f_{v}^{i_{s}} \rightarrow d f^{i_{1}} \wedge \cdots \wedge d f^{i_{s}} \quad \text { in } L^{\frac{\tilde{p}}{s}} \text { for } s=1, \ldots, k,
$$

and since $A_{i_{1} \cdots i_{k}} \in L^{\frac{\tilde{p}}{\tilde{p}-m}}$ and $g^{s} \in L^{\tilde{p}}, s=1, \ldots, m$, we then have by the Hölder inequality that

$$
\begin{aligned}
& A_{i_{1} \cdots i_{k}} \in L^{\frac{\tilde{p}}{\tilde{p}-k}} \\
& A_{i_{1} \cdots i_{k}} \wedge g^{i_{1}} \in L^{\frac{\tilde{p}}{\tilde{p}-(k-1)}} \longleftarrow \frac{\tilde{p}-k}{\tilde{p}-(k-1)}+\frac{1}{\tilde{p}-(k-1)}=1, \\
& A_{i_{1} \cdots i_{k}} \wedge g^{i_{1}} \wedge g^{i_{2}} \in L^{\tilde{\tilde{p}}-(k-2)} \longleftarrow \frac{\tilde{p}-k}{\tilde{p}-(k-2)}+\frac{1}{\tilde{p}-(k-2)}+\frac{1}{\tilde{p}-(k-2)}=1, \\
& \vdots \\
& A_{i_{1} \cdots i_{k}} \wedge g^{i_{1}} \wedge \cdots \wedge g^{i_{k-1}} \in L^{\frac{\tilde{p}}{\tilde{p}-1}} \longleftarrow \frac{\tilde{p}-k}{\tilde{p}-1}+\underbrace{\frac{1}{\tilde{p}-1}+\cdots+\frac{1}{\tilde{p}-1}}_{k-1}=1
\end{aligned}
$$

for $k=1, \ldots, m$.

Therefore, integrating both sides of (3.12) in $\Omega$, and then letting $v \rightarrow \infty$, we obtain

$$
\lim _{\nu \rightarrow \infty} I\left[F_{\nu}\right] \geq I[F]
$$

that is, we have

$$
\liminf _{v \rightarrow \infty} I\left[F_{\nu}\right]=\lim _{v \rightarrow \infty} I\left[F_{\nu}\right] \geq I[F]
$$

which is the desired result.

\section{Existence of minimizers}

To prove the existence of minimizers of $I$, another important point is requiring its coercivity condition. The counterpart in the variational problem of differential forms is introduced by Iwaniec and Lutoborski [1] as follows.

Definition 4.1 [1] Suppose that $W$ satisfies (1.1), we say that $W$ is coercive in the mean if

$$
\int_{\mathbb{R}^{n}} W\left(\zeta^{1}, \ldots, \zeta^{m}\right) \geq \rho \int_{\mathbb{R}^{n}}\left(\left|\zeta^{1}\right|^{p_{1}}+\cdots+\left|\zeta^{m}\right|^{p_{m}}\right)
$$

for all test forms $\zeta=\left(\zeta^{1}, \ldots, \zeta^{m}\right) \in L^{\mathbf{P}}\left(\mathbb{R}^{n}, \wedge^{l_{1}} \times \cdots \times \wedge^{l_{m}}\right)$. 
Theorem 4.2 In addition to the hypotheses, which ensure I to be weakly lower semicontinuous, assume that $W$ satisfies the growth condition (1.1) and mean-coercivity condition (4.1). Then the minimizer of $I$ is achieved at some $F \in \mathcal{M}$.

Proof Set

$$
m=\inf _{\mathcal{M}} I[F]
$$

It follows from (1.1) and (4.1) that $m$ is finite. By the definition of infimum, there exists a minimizing sequence $\left\{F_{v}\right\} \subset \mathcal{M}$ such that

$$
I\left[F_{v}\right] \rightarrow m
$$

as $v \rightarrow \infty$. Then the mean coerciveness (4.1) yields $\left\{F_{v}\right\}$ is bounded in $W^{1, \mathbf{p}}\left(\Omega, \wedge^{l_{1}-1} \times\right.$ $\left.\cdots \times \wedge^{l_{m}-1}\right)$, which implies that $\left\{f_{v}^{i}\right\}$ is bounded in $W^{1, p_{i}}\left(\Omega, \wedge^{l_{i}-1}\right), i=1, \ldots, m$. From Proposition 2.4, we see that $\left\{f_{v}^{i}\right\}$ has a weakly convergent subsequence, denoted by $\left\{f_{v}^{i}\right\}$ again, in $W^{1, p_{i}}\left(\Omega, \wedge^{l_{i}-1}\right)$. Then we may assume that

$$
f_{v}^{i} \rightarrow f^{i} \quad \text { in } W^{1, p_{i}}\left(\Omega, \wedge^{l_{i}-1}\right)
$$

as $v \rightarrow \infty$. Write $F=\left(f^{1}, \ldots, f^{m}\right)$, then we get

$$
F_{\nu} \rightarrow F \quad \text { in } W^{1, \mathbf{p}}\left(\Omega, \wedge^{l_{1}-1} \times \cdots \times \wedge^{l_{m}-1}\right)
$$

and $F \in \mathcal{M}$. Since $I$ is weakly lower semicontinuous, it follows that

$$
m \leq I[F] \leq \liminf _{\nu \rightarrow \infty} I\left[F_{\nu}\right]=m,
$$

i.e., $I[F]=m=\inf _{\mathcal{M}} I[F]$ as desired.

\section{Applications}

In this section, we present two examples to illustrate our main results. The first example is to explain the existence result Theorem 4.2 for the stored-energy function $W$, which is polyconvex.

Example 1 We consider a special case of $n=2, l=1, m=2$ and $g=0$. Let $\xi=\left(\xi^{1}, \xi^{2}\right) \in$ $\wedge^{1}\left(\mathbb{R}^{2}\right) \times \wedge^{1}\left(\mathbb{R}^{2}\right)$ with

$$
\xi^{1}=a_{1}^{1} d x^{1}+a_{2}^{1} d x^{2}, \quad \xi^{2}=a_{1}^{2} d x^{1}+a_{2}^{2} d x^{2}
$$

and assume that $W: \wedge^{1}\left(\mathbb{R}^{2}\right) \times \wedge^{1}\left(\mathbb{R}^{2}\right) \rightarrow \wedge^{2}\left(\mathbb{R}^{2}\right)$ is defined by

$$
W(\xi)=W\left(\xi^{1}, \xi^{2}\right)=|\xi|^{2}\left(*|\xi|^{2}-2 \xi^{1} \wedge \xi^{2}\right)
$$

where $*$ is the Hodge star duality operator.

Step 1. Direct computation implies

$$
|W(\xi)| \leq|\xi|^{2}\left(|\xi|^{2}+2\left|\xi^{1}\right|\left|\xi^{2}\right|\right) \leq 6\left(\left|\xi^{1}\right|^{4}+\left|\xi^{2}\right|^{4}\right),
$$


then we see that $W$ satisfies the growth condition (1.1) with $\mathbf{p}=\left(p_{1}, p_{2}\right)=(4,4)$. Thus, we have $\tilde{p}=p_{1}=p_{2}=4>m=2, \frac{\tilde{p}}{\tilde{p}-m}=2$ and $\mathcal{M}=W_{0}^{1,4}\left(\Omega, \wedge^{1} \times \wedge^{1}\right)$.

Step 2. Note that $\xi^{1} \wedge \xi^{2}=\left(a_{1}^{1} a_{2}^{2}-a_{2}^{1} a_{1}^{2}\right) d x^{1} \wedge d x^{2}$ and

$$
\begin{aligned}
a_{1}^{1} a_{2}^{2}-a_{2}^{1} a_{1}^{2} & \leq\left|a_{1}^{1}\right|\left|a_{2}^{2}\right|+\left|a_{2}^{1}\right|\left|a_{1}^{2}\right| \\
& \leq \frac{1}{2}\left(\left|a_{1}^{1}\right|^{2}+\left|a_{2}^{1}\right|^{2}\right)+\frac{1}{2}\left(\left|a_{1}^{2}\right|^{2}+\left|a_{2}^{2}\right|^{2}\right) \\
& =\frac{1}{2}\left(\left|\xi^{1}\right|^{2}+\left|\xi^{2}\right|^{2}\right)=\frac{1}{2}|\xi|^{2},
\end{aligned}
$$

then $2\left(a_{1}^{1} a_{2}^{2}-a_{2}^{1} a_{1}^{2}\right) * 1 \leq|\xi|^{2} * 1,{ }^{\text {a }}$ i.e.,

$$
2 \xi^{1} \wedge \xi^{2} \leq|\xi|^{2} * 1 .
$$

Hence we have $W \geq 0$.

Step 3. Applying Proposition 9.1 in [1], yields $W$ satisfies (4.1).

Step 4. Suppose that $\zeta=\left(\zeta^{1}, \zeta^{2}\right) \in W^{1,4}\left(\Omega, \wedge^{1} \times \wedge^{1}\right)$ and

$$
\begin{aligned}
& \zeta^{1}=b_{1}^{1} d x^{1}+b_{2}^{1} d x^{2}, \\
& \zeta^{2}=b_{1}^{2} d x^{1}+b_{2}^{2} d x^{2} .
\end{aligned}
$$

Let

$$
A=\left(\begin{array}{ll}
a_{1}^{1} & a_{2}^{1} \\
a_{1}^{2} & a_{2}^{2}
\end{array}\right), \quad B=\left(\begin{array}{ll}
b_{1}^{1} & b_{2}^{1} \\
b_{1}^{2} & b_{2}^{2}
\end{array}\right) .
$$

Then $|\xi|=|A|,|\zeta|=|B|$ and $\xi^{1} \wedge \xi^{2}=\operatorname{det} A * 1, \zeta^{1} \wedge \zeta^{2}=\operatorname{det} B * 1$. Thus, we have

$$
\begin{aligned}
W(\xi)-W(\zeta) & =\left(|\xi|^{4}-|\zeta|^{4}\right) * 1-2\left(|\xi|^{2} \xi^{1} \wedge \xi^{2}-|\zeta|^{2} \zeta^{1} \wedge \zeta^{2}\right) \\
& =(\mathcal{F}(A)-\mathcal{F}(B)) * 1,
\end{aligned}
$$

where $\mathcal{F}(X)=|X|^{4}-2|X|^{2} \operatorname{det} X$. Write

$$
A \otimes B=a_{1}^{1} b_{1}^{1}+a_{2}^{1} b_{2}^{1}+a_{1}^{2} b_{1}^{2}+a_{2}^{2} b_{2}^{2} .
$$

Then by applying

$$
\mathcal{F}(A)-\mathcal{F}(B) \geq 4\left(|B|^{2}-\operatorname{det} B\right) B \otimes(A-B)-2|B|^{2}(\operatorname{det} A-\operatorname{det} B)^{[1,6]},
$$

we get from (5.1) that

$$
\begin{aligned}
W(\xi)-W(\zeta)= & (\mathcal{F}(A)-\mathcal{F}(B)) * 1 \\
\geq & \left(4\left(|B|^{2}-\operatorname{det} B\right) B \otimes(A-B)-2|B|^{2}(\operatorname{det} A-\operatorname{det} B)\right) * 1 \\
= & 4\left(|B|^{2}-\operatorname{det} B\right)\left(b_{1}^{1}\left(a_{1}^{1}-b_{1}^{1}\right)+b_{2}^{1}\left(a_{2}^{1}-b_{2}^{1}\right)\right. \\
& \left.+b_{1}^{2}\left(a_{1}^{2}-b_{1}^{2}\right)+b_{2}^{2}\left(a_{2}^{2}-b_{2}^{2}\right)\right) * 1-2|\zeta|^{2}\left(\xi^{1} \wedge \xi^{2}-\zeta^{1} \wedge \zeta^{2}\right) .
\end{aligned}
$$


Observe that

$$
\begin{aligned}
* \zeta^{1} \wedge\left(\xi^{1}-\zeta^{1}\right) & =\left(\xi^{1}-\zeta^{1}\right) \wedge * \zeta^{1} \\
& =\left\langle\xi^{1}-\zeta^{1} \mid \zeta^{1}\right\rangle * 1 \\
& =\left(b_{1}^{1}\left(a_{1}^{1}-b_{1}^{1}\right)+b_{2}^{1}\left(a_{2}^{1}-b_{2}^{1}\right)\right) * 1 .
\end{aligned}
$$

Similarly, we have

$$
\begin{aligned}
* \zeta^{2} \wedge\left(\xi^{2}-\zeta^{2}\right) & =\left(\xi^{2}-\zeta^{2}\right) \wedge * \zeta^{2} \\
& =\left\langle\xi^{2}-\zeta^{2} \mid \zeta^{2}\right\rangle * 1 \\
& =\left(b_{1}^{2}\left(a_{1}^{2}-b_{1}^{2}\right)+b_{2}^{2}\left(a_{2}^{2}-b_{2}^{2}\right)\right) * 1
\end{aligned}
$$

By substituting (5.3) and (5.4) into (5.2), we obtain that

$$
\begin{aligned}
W(\xi)-W(\zeta) \geq & 4\left(|B|^{2}-\operatorname{det} B\right)\left(* \zeta^{1}\right) \wedge\left(\xi^{1}-\zeta^{1}\right) \\
& +4\left(|B|^{2}-\operatorname{det} B\right)\left(* \zeta^{2}\right) \wedge\left(\xi^{2}-\zeta^{2}\right) \\
& -2|\zeta|^{2}\left(\xi^{1} \wedge \xi^{2}-\zeta^{1} \wedge \zeta^{2}\right)
\end{aligned}
$$

Then we have from (5.5) that the corresponding terms in (3.10) are

$$
\begin{aligned}
& A_{1}(\zeta)=4\left(|B|^{2}-\operatorname{det} B\right)\left(* \zeta^{1}\right), \\
& A_{2}(\zeta)=4\left(|B|^{2}-\operatorname{det} B\right)\left(* \zeta^{2}\right)
\end{aligned}
$$

and

$$
A_{1,2}(\zeta)=2|\zeta|^{2}
$$

Since $\zeta=\left(\zeta^{1}, \zeta^{2}\right) \in W^{1,4}\left(\Omega, \wedge^{1} \times \wedge^{1}\right)$ and $\Omega$ is bounded, then we get that $A_{1}, A_{2}, A_{1,2} \in L^{2}$. Therefore, it follows from Theorem 4.2 that the minimizer of $I$ can be achieved.

Finally, we give an example for a stored-energy function $W$, which is quasiconvex.

Example 2 We consider the case $l=1, m=n$ and $g=0$. Let $\xi=\left(\xi^{1}, \ldots, \xi^{n}\right) \in \wedge^{1}\left(\mathbb{R}^{n}\right) \times$ $\cdots \times \wedge^{1}\left(\mathbb{R}^{n}\right)$ and

$$
\xi^{i}=a_{1}^{i} d x^{1}+\cdots+a_{n}^{i} d x^{n}
$$

for $i=1, \ldots, n$. Write $A=\left(a_{j}^{i}\right), 1 \leq i, j \leq n$, where $a_{j}^{i}$ denotes the element of $i$ th-row and $j$ th-column in $A$. Fix $1 \leq k \leq n$, then we have by simple calculation that

$$
\xi^{i_{1}} \wedge \cdots \wedge \xi^{i_{k}}=\sum_{1 \leq j_{1}<\cdots<j_{k} \leq n} \operatorname{det}\left(\begin{array}{ccc}
a_{j_{1}}^{i_{1}} & \ldots & a_{j_{k}}^{i_{1}} \\
\vdots & \ddots & \vdots \\
a_{i_{1}}^{i_{k}} & \ldots & a_{j_{k}}^{i_{k}}
\end{array}\right) d x_{j_{1}} \wedge \cdots \wedge d x_{j_{k}}
$$


for all ordered $l$-tuples $I=\left(i_{1}, \ldots, i_{k}\right), 1 \leq i_{1}<\cdots<i_{k} \leq n$. Let $J_{i_{1}, \ldots, i_{k}}=\{1, \ldots, n\}-I$, then it follows from (5.6) that

$$
\xi^{i_{1}} \wedge \cdots \wedge \xi^{i_{k}} \wedge d x_{I_{i_{1}, \ldots, i_{k}}}=\operatorname{det}\left(\begin{array}{ccc}
a_{i_{1}}^{i_{1}} & \ldots & a_{i_{k}}^{i_{1}} \\
\vdots & \ddots & \vdots \\
a_{i_{1}}^{i_{k}} & \ldots & a_{i_{k}}^{i_{k}}
\end{array}\right) d x .
$$

Here,

$$
A_{i_{1}, \ldots, i_{k}}^{i_{1}, \ldots, i_{k}} \triangleq \operatorname{det}\left(\begin{array}{ccc}
a_{i_{1}}^{i_{1}} & \ldots & a_{i_{k}}^{i_{1}} \\
\vdots & \ddots & \vdots \\
a_{i_{1}}^{i_{k}} & \ldots & a_{i_{k}}^{i_{k}}
\end{array}\right)
$$

denotes the $k \times k$ principal minors of $A$. Let $[A]_{k}$ denote the sum of the $k \times k$ principal minors of $A$, then

$$
\begin{aligned}
W(\xi) & \triangleq \sum_{k=1}^{n} \sum_{1 \leq i_{1}<\cdots<i_{k} \leq n} \xi^{i_{1}} \wedge \cdots \wedge \xi^{i_{k}} \wedge d x_{j_{i_{1}, \ldots, i_{k}}} \\
& =\sum_{k=1}^{n}[A]_{k} d x .
\end{aligned}
$$

Suppose that $A$ is positive semidefinite, then $W \geq 0$, and we have from [21] that $W$ is quasiconvex.

\section{Competing interests}

The authors declare that they have no competing interests.

\section{Authors' contributions}

All authors contributed equally to this paper. They read and approved the final manuscript.

\section{Acknowledgements}

This work was supported by the National Natural Science Foundation of China (Grant No. 11071048).

\section{Endnote}

a Inequality between two volume forms should be understood as inequality between their coefficients with respect to the standard basis, that is to say, we say that an $n$-form $\alpha$ on $\mathbb{R}^{n}$ is nonnegative if $\alpha=\lambda d x$ for some nonnegative function $\lambda$.

\section{Received: 6 March 2013 Accepted: 31 July 2013 Published: 22 August 2013}

\section{References}

1. Iwaniec, T, Lutoborski, A: Integral estimates for null Lagrangians. Arch. Ration. Mech. Anal. 125, 25-79 (1993)

2. Iwanice, T, Scott, C, Stroffolini, B: Nonlinear Hoge theory on manifolds with boundary. Ann. Mat. Pura Appl. 177 37-115 (1999)

3. Iwaniec, T, Martin, G: Geometric Function Theory and Nonlinear Analysis. Oxford University Press, New York (2001)

4. Chern, SS, Chen, WH: Lectures on Differential Geometry. Peking University Press, Beijing (2001)

5. Agarwal, RP, Ding, S, Nolder, C: Inequalities for Differential Forms. Springer, New York (2009)

6. Wang, T, Bao, G: Some properties of solutions to a class of Dirichlet boundary value problems. Abstr. Appl. Anal. (2012). doi:10.1155/2012/268612

7. Gilbarg, D, Trudinger, NS: Elliptic Partial Differential Equations of Second Order. Springer, New York (1983)

8. Heinonen, J, Kilpelainen, T, Martio, O: Nonlinear Potential Theory of Degenerate Elliptic Equations. Oxford University Press, Oxford (1993)

9. Dacorogna, B: Direct Methods in the Calculus of Variations. Springer, Berlin. Reprinted by World Publishing Corporation, Beijing (1991)

10. Morrey, CB: Multiple Integrals in the Calculus of Variations. Springer, Berlin (1966) 
11. Zhang, GQ: Lectures on Calculus of Variations. Higher Education Press, Beijing (2011)

12. Ball, JM: Convexity conditions and existence theorems in nonlinear elasticity. Arch. Ration. Mech. Anal. 63, 337-403 (1977)

13. Ball, JM, Currie, JC, Olver, PJ: Null Lagrangians, weak continuity, and variational problems of arbitrary order. J. Funct. Anal. 41, 135-174 (1981)

14. Ball, JM, Murat, F: $W^{1, p}$ Quasiconvexity and variational problems for multiple integrals. J. Funct. Anal. 58, 225-253 (1984)

15. Struwe, M: Variational Methods. Springer, Berlin (2008)

16. Yan, B: Introduction to variational methods in partial differential equations and applications. A summer course at Michigan State University (Math 890, Summer 2008)

17. Schmidt, B: Weak convergence methods for nonlinear partial differential equations. Lecture notes (Summer term, 2010)

18. Dacorogna, B, Marcellini, P: A counterexample in the vectorial calculus of variations. In: Material Instabilities in Continuum Mechanics, pp. 77-83. Oxford Science Publications, Oxford (1988)

19. Alibert, JJ, Dacorogna, B: An example of a quasiconvex function that is not polyconvex in two dimensions. Arch. Ration. Mech. Anal. 117, 155-166 (1992)

20. Šverák, V: New examples of quasiconvex functions. Arch. Ration. Mech. Anal. 119, 293-300 (1992)

21. Daniel, F, Xiao, Z: Quasiconvex functions and Hessian equations. Arch. Ration. Mech. Anal. 168, $245-252$ (2003)

22. Zhang, GQ, Lin, YQ: Lectures on Functional Analysis. Peking University Press, Beijing (2008)

doi:10.1186/1029-242X-2013-407

Cite this article as: Wang et al.: Direct methods in the calculus of variations for differential forms. Journal of Inequalities and Applications 2013 2013:407.

\section{Submit your manuscript to a SpringerOpen ${ }^{\circ}$ journal and benefit from:}

- Convenient online submission

Rigorous peer review

- Immediate publication on acceptance

- Open access: articles freely available online

- High visibility within the field

- Retaining the copyright to your article 